\title{
Vorwort zur Gesamtreihe
}

Die neuen Handbücher zur Wirtschaftsgeschichte setzen bei aktuellen Forschungen auf dem Gebiet der Wirtschaftsgeschichte an und richten sich auf spezifische Themenfelder aus. Damit unterscheiden sie sich von den bisherigen Handbüchern zur Wirtschaftsgeschichte, die einen eher chronologischen oder auch regionalen bzw. länderspezifischen Ansatz verfolgten und deren Erscheinen einige Jahrzehnte zurückliegt. Das Fach hat sich inzwischen weiter ausdifferenziert und dabei auch neue Themen und Methoden in den Blick genommen. Im Ganzen soll die neue Handbuchreihe eine vertiefte, sachbezogene Auseinandersetzung mit Schwerpunkten des Fachs auf der Basis einer breiten Wahrnehmung der Forschung ermöglichen. Sie richtet sich in erster Linie an Fachhistorikerinnen und Fachhistoriker, aber auch an Studierende, Lehrerinnen und Lehrer sowie an ein breites, historisch interessiertes Publikum.

Während für den englischsprachigen Raum vergleichbare Handbuchkonzepte für unterschiedliche Fächer und Forschungsfelder schon seit längerem vorliegen (Oxford und Cambridge Handbooks), ist dies für das Fach Wirtschaftsgeschichte im deutschsprachigen Raum nicht der Fall. Die vorliegende Handbuchreihe richtet sich dementsprechend in erster Linie am deutschsprachigen Forschungsraum aus, nicht ohne die jeweiligen Inhalte auch in einen internationalen bzw. transnationalen Kontext zu stellen.

Das übergeordnete Thema „Wirtschaft“ wird von uns in einem breiten Verständnis rezipiert und soll Anschlussmöglichkeiten an gesellschaftliche, politische, soziale und kulturelle Fragen bieten, ohne Vollständigkeit anstreben zu können. Die Beiträge der einzelnen Bände zeichnen sich durch eine inhaltliche und methodische Vielfalt aus, wobei die jeweilige Schwerpunktsetzung und Gestaltung in der Verantwortung der Herausgeber der Bände liegt.

Marcel Boldorf (Lyon)

Christian Kleinschmidt (Marburg) 
